\title{
Bursal immunopathology responses of specific-pathogen-free chickens and red jungle fowl infected with very virulent infectious bursal disease virus
}

\begin{abstract}
Very virulent infectious bursal disease virus (vvIBDV) targets B lymphocytes in the bursa of Fabricius (BF), causing immunosuppression and increased mortality rates in young birds. There have been few studies on the host immune response following vvIBDV infection at different inoculum doses in chickens with different genetic backgrounds. In this study, we characterized the immune responses of specific-pathogen-free (SPF) chickens and Malaysian red jungle fowl following infection with vvIBDV strain UPM0081 at 103.8 and 106.8 times the 50\% embryo infectious dose (EID50). The viral burden, histopathological changes, immune cell populations, and expression of immune-related genes were measured and compared between infected and uninfected bursa at specific intervals. The populations of KUL1+, $\mathrm{CD} 3+\mathrm{CD} 4+$ and $\mathrm{CD} 3+\mathrm{CD} 8+$ cells were significantly increased in both types of chickens at 3 $\mathrm{dpi}$, and there was significant early depletion of $\operatorname{IgM}+\mathrm{B}$ cells at $1 \mathrm{dpi}$ in the red jungle fowl. vvIBDV infection also induced differential expression of genes that are involved in Th1 and pro-inflammatory responses, with groups receiving the higher dose (106.8 EID50) showing earlier expression of IFNG, IL12B, IL15, IL6, CXCLi2, IL28B, and TLR3 at 1 dpi. Although both chicken types showed equal susceptibility to infection, the red jungle fowl were clinically healthier than the SPF chickens despite showing more depletion of IgM+ B cells and failure to induce IFNB activation. In conclusion, high-dose vvIBDV infection caused an intense early host immune response in the infected bursa, with depletion of IgM+ B cells, bursal lesions, and cytokine expression as a response to mitigate the severity of the infection.
\end{abstract}

Keyword: Very virulent infectious bursal disease virus (vvIBDV); Immune; Chicken 